tubes, but I have no doubt that a search into medical history could tell us. I remember, however, that the first Herible stethoscope which I ever saw was the one depicted in Fir, 6 , and used by Dr. Golding Bird when he saw outpatients in the year 1843. Being much crippled with rheumatism, and therefore not wishing to rise from bis chair, he found this instrument very convenient; he also was enabled to pass the ear-piece to gentlemen standing near him, whilst he held the cup on the part to be examined. I always thought it was his own invention. But, whether so or not, I do not think any great effort of genius was required to frame a flexible instrument, and then adapt it for the use of one or two ears. This being done, the next step would be to make two mouthpieces to apply to the chest at different spots. Various modifications of these instruments have been made of late years, but the first notice of them I have any knowledge of in my reading is to be found in a letter to THE LANCET of Aug. 29th, 1829, by Mr. Comins of Edinburgh, headed "A Flexible Stethoscope." This was only twelve years after Leannec's invention. It is difficult from his description to picture the instrument, but it seems to have been composed

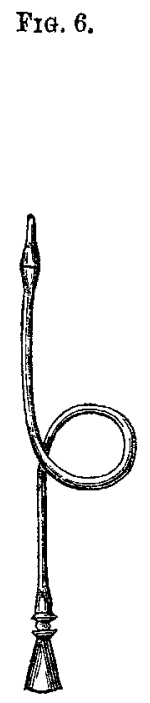

Fra. 7.

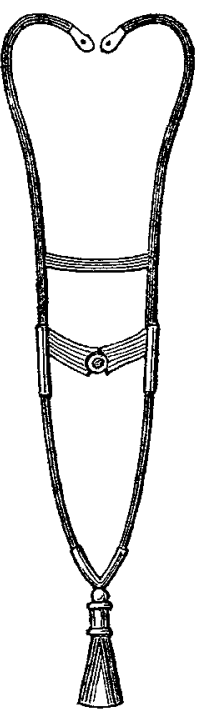

Fig. 8.

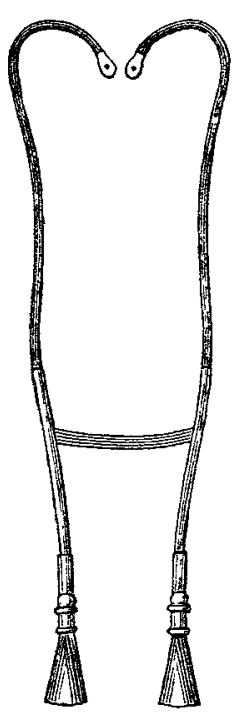

of jointed tubes, and made for two ears as well as one. Mr. Comins expresses his surprise that the discoverer of mediate auscultation did not suggest a flexible instrument, as he says "it can be used in the highest ranks of society without offending fastidious delicacy."

A very interesting fact was first pointed out to me by Dr. Andrew Clark, with respect to a peculiarity of the binaural in the objective appreciation of sounds; that if each ear piece be separately used, and any sound be made near themouth-piece, it is heard in the ear itself, but if the two pieces are employed together the sound is heard at the spot where it is produced. The fact is very interesting in a physiological point of view, and further corroborates the theory as to the value of a double set of senses, or, in a word, of the body being made up of two halves, for just as the two hands feeling different parts of an object gain an idea of extension, and the two eyes by obtaining different views of any substance get a knowlege of its solidity, so in the same way the two ears listening to the same sound more thoroughly appreciate its objectivity.

If you look at this series of drawings you may perceive but little resemblance between the first figure and the last, but take them one by one and you will see that the figures are really progressive. My story of development is not imaginary, but historical.

Aderdeen Medico-Chirurgical Society.-At the annual meeting of the Aberdeen Medico-Chirurgical Society, held on Thursday evening, the following officebearers were elected for the year:-President, Dr. John Trquhart; secretary and treasurer, Dr. James Rodger; librarian, William Frazer, surgeon; council, Drs. Beveridge, Garden, W. J. Simpson, Crombie, William Fraser ; representatives to the North of Scotland Medical Association, pre. sident and secretary ex officiis; Dr. Garden and Dr. J. C. $O$. will elected.

\section{NOTES ON CONCUSSION OF THE SPINAL CORD AND BRAIN.}

BY WM. SCOYELL SAFORY, F.R.S., SURGEOY TO ST. BARTHOLOMEW'S HOSPITAL.

Is the current view of the immediate cause of the paralysis which follows a severe injury, as a fracture, of some portion of the spine, correct, one? Can the loss, not only of volun. tary motion and sensation, but also of excito-motor reflex power in the part below, which is the usual effect, be directly due to pressure on some limited portion of the cord by the fractured bone? In experiments on animals, when the spinal cord is divided at any point, after the shock of the operation has passed off-and this is significant, - the power of reflex action in the part below, as everyone knows, remains. Why, then, is it abolished after fracture? Is it not that the violence which is sufficient to produce fracture is severe enough to produce such concussion or disturbance of the cord below as to destroy its power as a nerve centre? Instead of the common statement that the loss of reflex function is due to pressure at a particular part, would it not be more correct to say that the loss of reflex power below is the result of violence which at the part where it was directly applied was sufficient to break and displace the bone, but which has also injured the cord beyond? At all events, if the loss of reflex function be due to the fracture, it must be indirectly; to some mischief by displacement or dragging inflicted along the cord, of course far beyond the part directly implicated in the fracture; but, at least in many cases, the evidence strongly points to concussion as the cause. In a few exceptional cases, altbough compression of the cord by the displacement of the fractured bones continues, the portion below recovers its function as an independent centre, for the power of reflex action iu the lower limbs is, after a while, restored. The extension and persistence of mischief beyond the seat of visible injury surelv claim more consideration than they have hitherto received. But in any case it is not clear why the part above does not to a greater extent lose its reflex power. Une would imagine, I think, that the effect of such injury would usually extend almost equally, as indeed it occasionally does, in both directions. How is the clinical fact to be explained?

The curious fact that in concussion of the brain not only may there be complete loss of consciousness for a considerable period after the injury, but that in some cases after complete recovery there may be total loss of memory of all events which have happened shortly before the injury, bas not perhaps received the attention it deserves. The following cases may be taken as examples.

The driver of $a$ hansom cab was knocked off his box by an omnibus at the top of Newgate-street, and brought to the hospital in a state of complete unconsciousness from concussion. From this he speedily recovered; but, at least for several weeks after, when he appeared to be in every respect quite well, he could not in the slightest degree recollect what he had done or seen, or where he had been in the half hour immediately before the accident. It was known that within that time he had taken up a passenger at Cornbill, and driven along Cheapside and Newgate-street, yet of this he never had the faintest recollection ; but for all events up to this time his memory was excellent.

Again, a gentleman was thrown from his pony at polo, and picked up quite unconscious, in a state of concussion. He recovered from this in the course of two or three hours, and in a few days was as well as ever. But he could never recollect some striking events in the game which occurred within half an hour or so of the accident; yet up to this blank period his memory was minute and accurate.

Now, what happens in such a case? I suppose no one can tell. The substance of the brain is shaken, and presumably for a time damaged in some wav or disturbed; or, as we may say, its nutrition is temporarily impaired, and thus may subsequent loss of function be accounted for. The reco! lection of a bygone event shows that some impression which the brain receives at the time has been registered; and this must be, I suppose, by some change, however subtle, which is more or less durable. Does the violence of the concussion somehow interfere with the registration of 
such change; wiping out, as it were, the record before the ink is dry? But such guessing as this only shows how very far I am from understanding the fact.

\section{THE PATHOLOGY OF THE BLOOD IN INFLAMMATION.}

BY JAMES T. R. DAVISON, M.D. EDIN.,

SENIOR HOUSE-SURGEON, ROYAL SOUTHERN HOSPITAL, LIVERPOOL ; LATE RESIDENT PHYSICIAN, ROYAI INFIRMARY, EDINBURGH.

THE results obtained from the examination of some hundreds of specimens of pathological blood justify me in enunciating, as a pathological law, that "during the active period of inflammation the leucocytes of the blood are increased in numbers." The behaviour of the leucocytes under the inflammatory influence constitutes a most important help towards finding out the existence of inflammatory lesions. I need only mention those cases of obscure internal abscess and the first stages of pneumonia as examples of conditions where occasionally it is next to impossible to recognise them with certainty, and where the examination of the blood may lead us a good way on towards arriving at a certain diagnosis. This alteration of the blood is, for practical purposes, more important than the "hæmatoblastic crisis" described by M. Hayem, for while the latter comes into play at or about the time of the crisis of acute disorders, the former exhibits itself from the very first, even within a few hours from the commencement of the inflammatory process. Thus while the "hæematcblastic crisis" may for prognostic purposes be interesting, as indicating a favourable course of the disease, it possesses no diagnostic value; but the increase of leucocytes, by indicating the existence of an inflammatory process, and that from the very first, has a great diagnostic value, while it also is an element in prognosis, for the subsidence of the inflammation will be followed by a decrease of leucocytes.

It is well known that the leucocytes are found in greater numbers in the blood during the digestive process. How is this brought about? There is no necessity to assume that this increase is due to a more active formation of leucocytes in the spleen and other blood-forming organs. Recent pathology would favour the view that the increase of leucocytes in the blood is brought about reflexly by increased contraction of the splenic muscle and probably also of the lymphatic muscles, the point of origin of the reflex action being the gastro-intestinal canal. It has long been known that the spleen was capable of muscular contraction, and it has been left to Dr. Roy recently to discover that this contraction is rhythmical in time, and the directions through which nerve force influences this contraction. Dr. Roy believes that the spleen contains a nervous mechanism which regulates its contraction, but he has shown that this contraction is also affected by nerve influence from withoutfor example, stimulation of the peripheral end of a cut vagus causes contraction of the organ. ${ }^{1}$ It is therefore probable that the contraction of the gastric mussle during digestion stimulates the pneumogastric; that a nerve force is then carried to the medulla oblongata (Dr. Koy showed that stimulation of the vaso-motor centre in the medulla brought about contraction of the spleen), and thence is reflected through the vagi and splanchnics to the spleen, giving rise to increased contraction of its muscle, and thus to a discharge of leucocy tes into the circulation. These lencocytes having performed their function, whatever that may be, probably return into the spleen and lymphatic glands, as they are no longer found in excess in the blood after the digestive process is ended.

Once admitting that the spleen can be made to discharge its leucocy tes into the blood by a reflex action originating in the gastro-intestinal canal, and knowing the intricate and complicated connexions which exist in the sympathetic system, it will not be difficult to understand that the spleen can likewise be made to contract reflexly by a force originating in the arterioles and capillaries of any part of the body. In the inflammatory area a force-whether it be chemical, resulting from altered nutrition of the tissue cells,

1 Journal of Physiology, vol. iii., No. 3. oe nervous, reflected from a distant part-paralyses the vas? motor nerves of the arterioles. Either this same force, or one originating in the walls of the arterioles-the result of dis tension caused by increase of blood-pressure in the inflam. matory area-is carried to the medulla, and thence retlected to the spleen, and thus the spleen is advised that migration of leucocytes is taking place, and a call is made upon it to supply the demand made upon the blood. In inflammation there is therefore a loss of leucocytes from the blood, and an effort of nature to replace them, but, as in the case of byperprexia, nature over-does what is required of it; so here the supply from the spleen and lymphatic glands is greater than the demand, and therefore it is that during the active period of inflammation we find the leucocytes in the blood in such in. creased numbers.

The simplest form of inflammation that can be selected for examination of the changes in the blood is an abscess, for here there can be no suspicion of other influences being at work other than the inflammatory. If the abscess be acute and large the leucocytes are found in great abundance in the blood; but if the abscess be chronic, although it may be large in size, the leucocytes are not increased to such an extent. In the one case there is an active migration of leuco. cytes from the capillaries, and a consequent active demand upon the spleen and lymphatic glands; in the other case the migration is passive and small in quantity, and therefore the blood-forming organs are not called upon to supply a large amount of leucocytes. Small abscesses may also give rise to an abundant increase of leucocytes, and at other times to a slight increase only. Probably this difference depends upon the amount of tension and inflammatory fever present. In a case of abscess of the finger which had lasted for nine days, a small quantity of pus being present, but where the patient had passed sleepless nights, I found more leucocytes in the blood than in another case where a chronic abscess containing several ounces of pus had lasted for some months. After abscesses are opened the leucocytes in the blood begin to disappear, but sometimes the defervescence of these cells is interrupted by a secondary increase. When an abscess is opened tension is relieved, and the inflammatory process subdued, and the defervescence which then takes place is in accordance with the principle stated above, the demand for leucocytes being no longer present. The occasional secondary increase of these cells can easily be accounted for by an exacerbation of the inflammatory action. Next in importance to abscesses are scalds and burns, for in these we have an acute inflammation with visible effusion, which develops itself in a very short time, and thus we have an opportunity of examining the blood almost at the commence. ment of the inflammation. The leucocytes are always increased, and this increase I have noticed as early as two and $a$ half hours from the application of the irritant, clearly proving that the blood-forming organs have acted reflexly and emptied themselves of part of their contents, for in such a short time as two and a half hours the spleen and lym. phatics could not have formed all the extra leucocytes found in the blood. In cases of severe scalds and burns the excess of leucocytes may last for a long time, as a pus-secreting surface is left, and hence the migration of leucocytes continues. The same conditions exist in old discharing extensive surtaces, and the same excess of leucocytes is found in the blood. Again, the same principle is manifested in inflammation of serous membranes. In pleurisy the leucocytes may be present in very large numbers, diminis. ing with the lowering of the temperature. In pleurisy, as in other inflammations, a return of the temperature to normal is not immediately followed by a sudden and rapid decrease of the leucocytes. It is easier for the white corpuscles to enter the circulation than to leave it. Doubtless the lowering of the blood-pressu: which takes place at the crisis of an acute inflammation favours the return of the leucocytes from the blond, for the consequent retardation of the peripheral circulation is the nearest physiological approach to pathological stasis, an: thus the leucocytes are placed under the best possible circumstances to attach themselves to the capillary walls, an? eventually to regain the lymphatic system. Still this cos. not take place in a short time, and it is, therefore, that the inflammatory crisis is not immediately followed by a sudien and rapid decrease of the leucocytes. In peritonitis and acute buraitis I found the white corpuscles in excess. In a case of double effusion into the knee-joints four dars with a temperature of $1004^{\circ}$, I did not find an increase the white corpuscles, and here I may state that wr ex 